\title{
Heparina e insulina en el manejo de pancreatitis aguda asociada a hipertrigliceridemia
}

\section{Heparin and insulin in the management of acute hypertriglyceridemic pancreatitis}

\author{
Juan Pablo Camargo-Mendoza, Daniel Efrén Rodríguez-Ariza, Jhonny Steven Bustos-Calvo \\ - Bogotá, D.C. (Colombia)
}

DOI: https://doi.org/10.36104/amc.2020.1491

\begin{abstract}
Resumen
La pancreatitis secundaria a hipertrigliceridemia (PASHT) es la tercera causa de pancreatitis aguda en la mayoría de series, teniendo como factor de riesgo un valor de triglicéridos (TG) mayor a $1000 \mathrm{mg} / \mathrm{dL}$. El mecanismo fisiopatológico involucra la hidrólisis de triglicéridos por la lipasa pancreática y la liberación de ácidos grasos que inducen el daño por la generación de radicales libres. La reducción de los TG a niveles menores de $500 \mathrm{mg} / \mathrm{dL}$ es el objetivo del tratamiento, basado en el aumento de la actividad de la lipoproteinlipasa y degradación de quilomicrones. Presentamos un caso de un paciente que presenta PASHT con adecuada respuesta al manejo concomitante de insulina y heparina. (Acta Med Colomb 2020; 45. DOI: https://doi.org/10.36104/amc.2020.1491).
\end{abstract}

Palabras clave: triglicéridos, pancreatitis aguda, heparina, insulina, lipoproteína lipasa.

\begin{abstract}
Hypertriglyceridemic pancreatitis (HTGP) is the third cause of acute pancreatitis in most studies, with a triglyceride (TG) risk value of more than $1000 \mathrm{mg} / \mathrm{dL}$. The pathophysiological mechanism involves triglyceride hydrolysis by pancreatic lipase and the release of fatty acids which cause damage by producing free radicals. The reduction of TGs below $500 \mathrm{mg} / \mathrm{dL}$ is the treatment goal, based on increased lipoprotein lipase activity and chylomicron degradation. We present the case of a patient with HTGP with an adequate response to concomitant insulin and heparin therapy. (Acta Med Colomb 2020; 45. DOI: https://doi.org/10.36104/amc.2020.1491).

Key words: triglycerides, acute pancreatitis, heparin, insulin, lipoprotein lipase.
\end{abstract}

Dr. Juan Pablo Camargo-Mendoza: Médico Internista, Neumólogo y Epidemólogo Clínico, Magister en Hipertensión Pulmonar, Unidad de Cuidado Intensivo Hospital de Kennedy; Dr. Daniel Efrén Rodríguez-Ariza: Médico Internista, Magister en Epidemiología, Unidad de Cuidado Intensivo Hospital de Kennedy; Dr. Jhonny Steven Bustos-Calvo: Cirujano General, Servicio de Cirugía Hospital de Kennedy. Bogotá, D.C. (Colombia)

Correspondencia: Dr. Juan Pablo CamargoMendoza. Bogotá, D.C. (Colombia). E-mail: jpcamargome@unal.edu.co Recibido: 27/VIII/2019 Aceptado: 5/II/2020

\section{Introducción}

La pancreatitis aguda es una inflamación del parénquima glandular del órgano retroperitoneal, que conlleva a injuria con o sin subsecuente destrucción del acino pancreático (1). La incidencia de pancreatitis varía según la población (4.9-35 por cada 100000 habitantes). El promedio de edad de presentación es 53 años con distribución equivalente entre géneros, aunque en los últimos años existe un incremento en mujeres menores de 35 años. El promedio de estancia hospitalaria es siete días, lo que sugiere que la mayoría de los casos son leves; sin embargo, uno de cada cinco casos pueden desarrollar daño de órgano, con o sin complicaciones definiéndolos como casos severos. Paciente con pancreatitis severa que supera la primera fase de la enfermedad puede desarrollar infección secundaria en el páncreas necrótico. La mortalidad en paciente con necrosis infectada o falla de órgano se ha determinado en 30-40\% lo cual aumenta con el incremento de la edad (2).

Cálculos biliares es la causa más común constituyendo el $40-70 \%$ de los casos. Estudios poblacionales demuestran que $3-7 \%$ de la personas con cálculos biliares desarrollan pancreatitis. Ingesta de alcohol es la segunda causa más común con $25-41 \%$. Hipertrigliceridemia es la tercera causa y puede llegar a $10 \%$ de todos los casos, incrementándose hasta 50\% en el embarazo. Las tasas de severidad y complicaciones tienden a ser mayores (3), por tal motivo 
el manejo médico oportuno con disminución de los TG es fundamental, el siguiente caso muestra una forma de manejo que es considerada costo efectiva.

\section{Presentación del caso}

Paciente de 30 años que ingresó por cuadro de dos días de evolución de dolor en epigastrio irradiado a región esternal y precordial, emesis en número de 10 de contenido alimentario, niega fiebre. Ingresa al servicio de urgencias deshidratado, taquicárdico, cifras tensionales normales, al examen físico no dolor a la palpación abdominal con examen neurológico sin alteraciones, glucometría de ingreso $352 \mathrm{mg} / \mathrm{dL}$, gases arteriales con acidosis metabólica parcialmente compensada sin trastorno de la oxigenación (ph 7.25 $\mathrm{HCO}_{3} 14 \mathrm{PCO}_{2} 29 \mathrm{PO}_{2} 63 \mathrm{BE}-10$ ), se toman paraclínicos que muestran hemograma dentro de los límites normales. Amilasa de $648 \mathrm{U} / \mathrm{L}$, hiponatremia de $120 \mathrm{mmol} /$ dL (corregido a la glucosa $124 \mathrm{mmol} / \mathrm{dL}$ ) y hemoglobina glicosilada de $14.10 \%$. Se inicia manejo con cristaloides en el contexto de cetoacidosis diabética (debuta la enfermedad) se realiza reanimación con cristaloides y se inicia manejo con insulina en infusión. Se diagnosticó de igual forma pancreatitis Marshall 2, APACHE II 10. Ecografía hepatobiliar descarta enfermedad litiásica, por lo que se solicitan triglicéridos en vista de no antecedente de consumo de alcohol, reportando un valor de $6700 \mathrm{mg} / \mathrm{dL}$, y colesterol total de $1071 \mathrm{mg} / \mathrm{dL}$. No disponibilidad de plasmaféresis por lo cual se considera asociación al manejo de insulina, heparina no fraccionada a dosis de 80 unidades $/ \mathrm{kg}$ en bolo y se continuó a $18 \mathrm{U} / \mathrm{kg} /$ hora. Tomografía de abdomen compatible con Baltazar B (Figura 1). Se corrige acidosis metabólica y se logra adecuado control glicémico, se continúa dextrosa, insulina y heparina en infusión esta última manteniendo PTT 2.5 veces del valor de referencia de la institución. Paciente con mejora clínica de dolor abdominal y se realiza seguimiento diario de triglicéridos mostrando descenso significativo. Paciente tolera adecuadamente la vía oral, se inició fenofibrato como terapia complementaria. Terapia en infusión se mantuvo cuatro días, control

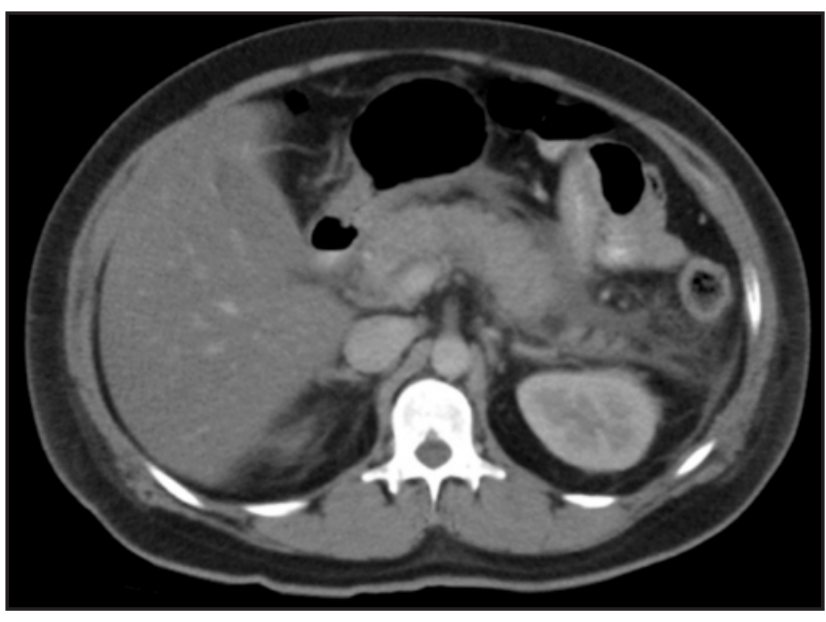

Figura 1. Aumento difuso del tamaño del páncreas, con contornos irregulares y atenuación heterogénea.

de triglicéridos al quinto día por debajo de $500 \mathrm{mg} / \mathrm{dL}$, se traslada a piso sin complicaciones (Tabla 1).

\section{Discusión}

La PASHT es una enfermedad que se presenta en personas con desórdenes del metabolismo de los lípidos, principalmente en aquellos que tienen niveles de triglicéridos superiores a $1000 \mathrm{mg} / \mathrm{dL}$; se presenta generalmente en hombres y en la quinta década de la vida (46.9 \pm 11.5 años) (4).

El mecanismo fisiopatológico no está bien definido, pero se reconocen tres teorías; la primera, es la más aceptada y propone que los quilomicrones ocluyen los capilares pancreáticos, la hidrólisis de éstos libera altas concentraciones de ácidos grasos libres que sobrepasan la capacidad de la albúmina para agregarla en micelios con propiedades detergentes. Estos complejos ácidos grasos-micelios van a generar lesión endotelial y de las células acinares del páncreas $(5,6)$, la isquemia que resulta crea un ambiente ácido el cual genera más ácidos grasos libres y toxicidad, además de llevar a la liberación y activación de lipasa pancreática y proteasas y lesión secundaria a autoingestión (4). La

Tabla 1. Registro de laboratorios.

\begin{tabular}{|c|c|c|c|c|c|}
\hline & Dia 1 & Dia 2 & Dia 3 & Dia 4 & Dia 5 \\
\hline Triglicéridos mg/dL & 6700 & 3250 & 1770 & 749 & 454 \\
\hline Colesterol total mg/dL & 1071 & 850 & 577 & 435 & 325 \\
\hline Leucocitos células $/ \mu \mathrm{L}$ & 14580 & 10510 & 8530 & 9550 & 7520 \\
\hline $\mathrm{Hgb} \mathrm{g} / \mathrm{dL}$ & 16.2 & 15 & 12.3 & 12.5 & 13 \\
\hline Hematocrito \% & 40 & 41 & 36 & 37 & 37.9 \\
\hline Plaquetas células $/ \mu \mathrm{L}$ & 302000 & 310000 & 226000 & 297000 & 206200 \\
\hline Sodio $\mathrm{mmol} / \mathrm{L}$ & 128 & 132 & 136 & 135 & 138 \\
\hline Potasio $\mathrm{mmol} / \mathrm{L}$ & 3.32 & 3.5 & 3.58 & 3.78 & 3.65 \\
\hline
\end{tabular}


segunda teoría habla de la hiperviscosidad, la cual lleva a isquemia selectiva de las células acinares pancreáticas; la tercera es la teoría genética, la cual se basa en polimorfismos genéticos que han sido identificados en el paciente con hipertrigliceridemia severa con pancreatitis; los cuales no han sido encontrados en personas con hipertrigliceridemia sin pancreatitis (4).

El curso clínico de la PASHT es similar al de otras causas de pancreatitis aguda pero la tasa de complicaciones es significativamente más alta al igual que la mortalidad (4), durante la presentación clínica se describen generalmente cuatro grupos de personas PASHT: en primer lugar paciente con diabetes mal controlada (en el caso debutó con diabetes) con o sin historia de hipertrigliceridemia. En segundo lugar, paciente alcohólico con plasma lechoso. Tercero paciente sin obesidad, sin diabetes, no alcohólico con hipertrigliceridemia secundaria a fármacos o dieta y ultimo paciente con hiperlipidemia familiar sin un factor secundario (5).

El tratamiento agudo (primeros 14 días) es la clave del manejo ya que $50 \%$ de la mortalidad ocurre en este tiempo (posiblemente por inflamación sistémica y falla multiorgánica) y es similar al manejo de pancreatitis aguda de otras causas; el manejo consiste en: reposo intestinal con suspensión de la vía oral, reanimación hídrica endovenosa, control del dolor y manejo sintomático; la administración de cristaloides se debe realizar con líquidos isotónicos a una infusión de $250-500 \mathrm{~mL} / \mathrm{h}$ por $24-48$ horas. La nutrición enteral juega un papel importante en el manejo de la pancreatitis aguda, ofreciendo soporte nutricional al paciente manteniendo así la función intestinal y brindando protección contra el síndrome de respuesta inflamatoria sistémica (3). En el momento no hay un tratamiento específico para la hiperlipidemia, pero diferentes modalidades terapéuticas como la administración de insulina, heparina, aféresis/plasmaféresis, fibratos y ácidos grasos omega 3 se han implementado para reducir los niveles séricos de TG.

Recalcamos la importancia de la lipoproteína lipasa (LPL) una enzima que se expresa en las células endoteliales capilares del tejido muscular y del tejido adiposo cumpliendo un rol importante en el metabolismo graso hidrolizando los TG, siendo importante en la reducción de los niveles de los mismos. La insulina promueve la síntesis y activación de esta enzima reduciendo los niveles de TG e incrementa la degradación de quilomicrones, ésta puede ser administrada vía subcutánea o parenteral, aunque es preferible el uso parenteral por su facilidad de titulación y farmacocinética. Se utiliza en diferentes esquemas de infusión dependiendo de si el paciente tiene diabetes, logrando disminuir el nivel de triglicéridos a menos de 1000 $\mathrm{mg} / \mathrm{dL}$ en un periodo de 72-96 horas de infusión, en el caso en mención se utiliza de inicio por cetoacidosis diabética.

En una serie de casos (10 pacientes) el uso de insulina endovenosa se asoció con descenso de $40 \%$ de los TG en 24 horas en hipertrigliceridemias severas y un descenso de $87 \%$ en los pacientes tratados con insulina más ayuno, mientras que la administración subcutánea de insulina generó una reducción de $23 \%$ de los triglicéridos en 24 horas (6). La terapia con insulina ha mostrado ser segura y efectiva en el tratamiento de PASTH (4.5).

En cuanto la heparina, un medicamento ampliamente usado por su efecto anticoagulante en múltiples patologías, en el contexto de PASTH ha demostrado que altera el proceso inflamatorio por su efecto en las células endoteliales, bloqueando la adhesión plaquetaria y de leucocitos al endotelio y reduciendo el reclutamiento leucocitario, llevando a la inhibición de la reacción inflamatoria (7) llegando a demostrar una reducción en la mortalidad y mejoría del score de Balthazar, en un estudio multicéntrico realizado por Lu XS. (estudio con poder estadístico limitado) (7); además presenta al igual que la insulina un efecto sobre la LP activándola, la heparina genera la liberación de la LP endotelial a la circulación (6), llevando a un descenso de los TG séricos. El proceso posterior a la liberación de la LPL endotelial requiere de nuevo la síntesis de la misma, siendo este proceso lento, por lo que se presentan dos sucesos: el primero, es la pérdida de efectividad posterior a la primera dosis; el segundo, una hipertrigliceridemia de rebote que se puede presentar después de la descontinuación de heparina, por lo que se recomienda usar heparina posterior al manejo estándar basado en suspensión de la vía oral, administración de líquidos endovenosos, e infusión de insulina (9); por lo anterior la heparina no sería una terapia de elección como monoterapia (6). La administración de heparina puede realizarse subcutánea o parenteral, esta última en infusión o por bolos, no hay evidencia suficiente para dar una recomendación clara al respecto, pero según los reportes de casos se podría recomendar la administración parenteral en bolos siendo esta más efectiva (18 unidades/ kg dosis cada 4-6 horas) (6), además se debe considerar el riesgo de sangrado aunque éste no ha sido reportado en la literatura en pacientes con PASTH.

En cuanto el uso de insulina y heparina concomitante destacamos lo reportado por Henzen y cols donde el rango de disminución se encontraba entre $3822 \mathrm{mg} / \mathrm{dL}$ a $888 \mathrm{mg} /$ dL en 2.8 días con la administración simultánea de estos medicamentos en cinco pacientes (10). En la serie de cuatro casos de Kuchay y cols la disminución media fue de 3800 $\mathrm{mg} / \mathrm{dL}$ a $849.7 \mathrm{mg} / \mathrm{dL}$ en un tiempo de 72 horas, en todos los casos hubo resolución de la pancreatitis (11). Berger y cols en su serie de cinco casos los TG se encontraban por debajo de $500 \mathrm{mg} / \mathrm{dL}$ a los tres días (12). En nuestro caso hubo disminución de $3450 \mathrm{mg} / \mathrm{dL}$ en las primeras 24 horas y se lograron niveles menores de $500 \mathrm{mg} / \mathrm{dL}$ hasta el quinto día; sin embargo, el valor de inicio supera a muchos de los casos reportados en la literatura.

Se ha reportado el uso del intercambio de plasma terapéutico principalmente en pacientes con niveles de TG superiores a $2000 \mathrm{mg} / \mathrm{dL}$, presentando una rápida mejoría clínica no sólo por su efecto en el descenso de TG sino también por su 
efecto en los mediadores inflamatorios (4), la realización de una sesión de plasmaféresis ha demostrado que en pacientes con hipertrigliceridemia severa se genera un descenso de los TG séricos de hasta 70\% (13), y en pacientes con pancreatitis por hipertrigliceridemia se demostró un descenso superior a $80 \%$ en dos sesiones de plasmaféresis (14).

En el año 2016 He WH y cols realizaron un ensayo aleatorizado controlado con 66 pacientes con PASTH, encontrando que el uso temprano de hemofiltración de alto volumen puede disminuir los niveles de TG pero no es superior a la terapia basada en insulina y heparina de bajo peso molecular en cuanto a complicaciones pancreáticas locales, requerimiento de intervención quirúrgica, mortalidad y duración de la hospitalización (15).

\section{Conclusión}

El manejo con insulina y heparina es una alternativa en el tratamiento de la PASTH, segura y económica, comparado con la plamaféresis la cual no esta disponible en muchas instituciones. No obstante la mayoría de la literatura disponible se ha basado en series de casos por lo que se requieren estudios posteriores con mejor poder estadístico.

\section{Referencias}

1. Hammad AY, Ditillo M, Castanon L. Pancreatitis. Surg Clin N Am 2018; 98: 895-913.

2. Yadav D, Lowenfels AB. Trends in the epidemiology of the first attack of acute pancreatitis : a systematic review. Pancreas 2006; 33: 323-30.

3. Waller A, Long B, Koyfman A, Gottlieb M. Acute Pancreatitis: Updates for Emergency Clinicians. J Emerg Med 2018 ;55:769-779.

4. Adiamah A, Psaltis E, Crook M, Lobo DN. A systematic review of the epidemi- ology, pathophysiology and current management of hyperlipidaemic pancreatitis. Clin Nutr 2018;37:1810-22.

5. Senosiain Lalastra C, Tavío Hernández E, Moreira Vicente V, Maroto Castellanos M, García Sánchez MC, Aicart Ramos M, et al. Pancreatitis aguda por hipertrigliceridemia. Gastroenterol Hepatol 2013;36:274-9.

6. Rawla P, Sunkara T, Thandra KC, Gaduputi V. Hypertriglyceridemia-induced pancreatitis: updated review of current treatment and preventive strategies. Clin J Gastroenterol 2018;11:441-8.

4. Kuchay MS, Farooqui KJ, Bano T, Khandelwal M, Gill H, Mithal A. Heparin and insulin in the management of hypertriglyceridemia-associated pancreatitis: case series and literature review. Arch Endocrinol Metab 2017;61:198-201.

5. Thuzar M, Shenoy VV, Malabu UH, Schrale R, Sangla KS. Extreme hypertriglyceridemia managed with insulin. J Clin Lipidol 2014;8:630-4.

6. Trzaskoma A, Kruczek M, Rawski B, Poniewierka E, Kempiński R. The use of heparin in the treatment of acute pancreatitis. Pol J Surg 2013;85:223-7

8. Lu X-S, Qiu F, Li J-Q, Fan Q-Q, Zhou R-G, Ai Y-H, et al. Low molecular weight heparin in the treatment of severe acute pancreatitis: a multiple centre prospective clinical study. Asian J Surg 2009;32:89-94.

9. Mike M. Mechanism for Heparin's Ability to Lower TGs in Hypertriglyceridemia [Internet]. 2015. https://www.ebmconsult.com/articles/heparin-mechanismreduce-triglycerides-hypertriglyceridemia-treatment

10. Henzen C, Rock M, Schnieper C, Heer K. Heparin and insulin in the treatment of acute hypertriglyceridemia-induced pancreatitis. Schweiz Med Wochenschr. 1999;129:1242-8.

11. Kuchay MS, Farooqui KJ, Bano T, Khandelwal M, Gill H, et al. Heparin and insulin in the management of hypertriglyceridemia-associated pancreatitis: case series and literature review. Arch Endocrinol Metab 2017;61:198-201.

12. Berger Z, Quera R, Poniachik J, Oksenberg D, Guerrero J. [Heparin and insulin treatment of acute pancreatitis caused by hypertriglyceridemia. Experience of 5 cases]. Rev Med Chil. 2001;129:1373-8.

13. Ewald N, Kloer H-U. Severe hypertriglyceridemia: An indication for apheresis? Atheroscler Suppl 2009;10:49-52.

14. Yeh J-H, Chen J-H, Chiu H-C. Plasmapheresis for hyperlipidemic pancreatitis. J Clin Apheresis. 2003;18:181-5.

15. He W, Yu M, Zhu Y, Xia L, Liu P, Zeng H, et al. Emergent triglyceride-lowering therapy with early high-volume hemofiltration against low-molecular-weight heparin combined with insulin in hypertriglyceridemic pancreatitis: A prospective randomized controlled Trial. J Clin Gastroenterol. 2016;50:772-8. 